\title{
ON COMPACT HAUSDORFF SPACES OF COUNTABLE TIGHTNESS
}

\author{
ZOLTÁN BALOGH
}

(Communicated by Dennis Burke)

\begin{abstract}
A general combinatorial theorem for countably compact, noncompact spaces is given under the Proper Forcing Axiom. It follows that compact Hausdorff spaces of countable tightness are sequential under PFA, solving the Moore-Mrowka Problem. Other applications are also given.
\end{abstract}

\section{INTRODUCTION}

The subject matter of this paper is the following old problem of set-theoretic topology [MM]:

Moore-Mrowka Problem. Is every compact Hausdorff space of countable tightness sequential?

A space $X$ is said to be of countable tightness if the closure of every set $A$ in $X$ equals the union of the closures of all countable subsets of $A . X$ is said to be sequential if for every nonclosed subset $A$ there is countable sequence of points of $A$ that converges to a point outside $A$.

In many models of ZFC, Zermelo Fraenkel Set Theory with the axiom of Choice, it was shown that the answer to the Moore-Mrowka Problem is no. Counterexamples were constructed by Osztaszewski [O] and Fedorchuck [F] from the set-theoretic principle $\diamond$.

The existence of counterexamples is compatible with Martin's Axiom together with the negation of $\mathrm{CH}$; and it also is compatible with the Proper Forcing Axiom restricted to posets of size $\leq \omega_{1}$ (Nyikos $\left[\mathrm{Ny}_{2}\right]$ ).

In the other direction, several partial results were proven. In particular, $\mathrm{MA}+\neg \mathrm{CH}$ implies that the answer to the Moore-Mrowka Problem is yes in the class of hereditarily separable spaces (Szentmiklóssy [Sz]), and the Proper Forcing Axiom implies that the answer is yes for hereditarily normal spaces (Nyikos $\left[\mathrm{Ny}_{3}\right]$ ).

Received by the editors January 27, 1987 and, in revised form, August 19, 1987.

1980 Mathematics Subject Classification (1985 Revision). Primary 54A35, 54D30, 03E35; Secondary 54D45.

Key words and phrases. Proper Forcing Axiom, countably compact, compact Hausdorff, countable tightness, sequential, perfect preimage of $\omega_{1}$. 
A more detailed treatment of the Moore-Mrowka Problem and related questions can be found in the problem section of [TP], Classic Problem VI, and in $\left[A_{3}, \S 2.3\right]$. The problem was repeatedly mentioned in $\left[A_{1}, A_{2}, A_{3}\right]$.

In this paper we shall prove that Shelah's Proper Forcing Axiom (PFA) implies that the answer to the Moore-Mrowka Problem is yes. We shall actually prove a general combinatorial theorem (Theorem 1.1) from which an affirmative answer to the Moore-Mrowka Problem follows. Theorem 1.1 has other applications; some of these will also be included.

Our approach to PFA owes much to Fremlin's version $\left[F R_{1}, F_{2}\right.$ ] of a method of Todorcevic [T]. Based on these works of Fremlin, significant progress concerning topological applications of PFA has been made by Nyikos and Fremlin (see in $\left[\mathrm{NY}_{3}, \mathrm{NY}_{4}, \mathrm{FR}_{1}, \mathrm{FN}\right]$ ) part of which is related to this paper.

Throughout the paper we use current terminology of set theory and settheoretic topology as is used in $[\mathrm{K}]$ and $[\mathrm{KV}]$, e.g. In particular, cardinals are initial ordinals, ordinals are ordered by $\in$, and $[A]^{\leq \kappa}=\left\{A^{\prime} \subseteq A:\left|A^{\prime}\right| \leq \kappa\right\}$. If $A$ is a subset of a topological space $X$, then the closure of $A$ in $X$ will be denoted by $\bar{A}$ everywhere except in the proof of Theorem 2.1, where the notation $\mathrm{cl}_{X}(A)$ will be adopted for the sake of unambiguity.

\section{INCREASING $\omega_{1}$-SEQUENCES FOR SMALL RELATIONS}

Let $X$ be a nonvoid set, and let $A, V \subseteq X^{2}$ be reflexive relations on $X$. We shall say that a sequence $\left\{x_{\xi}: \xi \in \omega_{1}\right\}$ of distinct points of $X$ is an increasing $\omega_{1}$-sequence for the pair $\langle A, V\rangle$ if for every $\zeta \in \omega_{1}$,

$$
A\left(x_{\zeta}\right) \cap\left\{x_{\xi}: \xi \in \omega_{1}\right\} \subseteq\left\{x_{\xi}: \xi \leq \zeta\right\}
$$

and

$$
V\left(x_{\zeta}\right) \cap\left\{x_{\xi}: \xi \in \omega_{1}\right\} \supseteq\left\{x_{\xi}: \xi \leq \zeta\right\}
$$

hold. If $X$ is a topological space and $\mathscr{F}$ is a closed filter on $X$, then we shall say that a subset $Y$ of $X$ is $\mathscr{F}$-small if there is an $F \in \mathscr{F}$ with $Y \cap F=\varnothing$. A relation $A \subseteq X^{2}$ will be called $\mathscr{F}$-small-valued, if $A(x)$ is $\mathscr{F}$-small for every $x \in X$.

Now we can state the following theorem on the existence of $\omega_{1}$-sequences.

Theorem 1.1 (PFA). Let $X$ be a countably compact, noncompact $T_{1}$-space, and let $\mathscr{F}$ be a free closed filter on $X$. Further, suppose that $A, V \subseteq X^{2}$ is a pair of reflexive relations on $X$ such that $A$ is $\mathscr{F}$-small-valued and for every $x \in X$, $A(x)$ is closed and $V(x)$ is open.

Then there is an increasing $\omega_{1}$-sequence for the pair $\langle A, V\rangle$.

Proof. Let us note first that the conclusion of our theorem (that there is an increasing $\omega_{1}$-sequence for $\left.\langle A, V\rangle\right)$ depends neither on the topology of $X$ nor on the filter $\mathscr{F}$.

Let us thus consider a new topology $\tau^{\prime}$ on $X$ defined by the closure operation

$$
\mathrm{cl}_{\tau^{\prime}}(E)=\bigcup\left\{\bar{D}: D \in[E]^{\leq \omega}\right\}
$$


where $\bar{D}$ denotes closure of $D$ in the original topology $\tau .\left\langle X, \tau^{\prime}\right\rangle$ clearly has countable tightness. Since $\tau^{\prime}$ refines $\tau$, it follows that $\left\langle X, \tau^{\prime}\right\rangle$ is a $T_{1}$-space, $\mathscr{F}$ is a free closed filter-base in $\left\langle X, \tau^{\prime}\right\rangle$, and for every $x \in X, A(x)$ is closed and $V(x)$ is open in $\left\langle X, \tau^{\prime}\right\rangle .\left\langle X, \tau^{\prime}\right\rangle$ is countably compact, because a countably infinite closed discrete subspace in $\left\langle X, \tau^{\prime}\right\rangle$ would have the same properties in $\langle X, \tau\rangle$.

Further, if we extend $\mathscr{F}$ to a closed ultrafilter $\mathscr{F}^{\prime}$ in $\left\langle X, \tau^{\prime}\right\rangle$, then $\mathscr{F}$-small sets remain small with respect to $\mathscr{F}^{\prime}$, too.

So, if for given $A, V$ we can find $X$ and $\mathscr{F}$ satisfying the conditions of our theorem, then $X$ can be chosen countably tight and $\mathscr{F}$ can be chosen to be a closed ultrafilter.

Therefore, for the rest of the proof, we may assume that $X$ is of countable tightness and $\mathscr{F}$ is a free closed ultrafilter in $X$.

Let us set $\mathscr{H}=\{H \subset X$ : there is an $F \in \mathscr{F}$ with $H \cap F=\varnothing\}$.

Since $X$ is countably compact it follows that $\mathscr{H}$ is a proper $\sigma$-ideal on $X$. Let $\mathscr{H}_{0}$ be the family of open members of $\mathscr{H}$. Note that every member of $\mathscr{H}$ is contained in a member of $\mathscr{H}_{0}$. Also note that for $Z \subseteq X, Z \notin \mathscr{H}$ implies $X-\bar{Z} \in \mathscr{H}$.

Now let us define a partially ordered set $\langle T, \preceq\rangle$ in the following way. First, let $T$ be the set of all finite partial functions $t$ from $\omega_{1}$ to $X$ such that for every $\eta, \xi \in \operatorname{dom}(t), \eta<\xi, t(\xi) \notin A(t(\eta))$ holds. If $t \in T, \xi \in \operatorname{dom}(t)$, then an ordinal $\eta \in \omega_{1}$ will be called $(\xi, t)$-bad, if $\eta \in \operatorname{dom}(t) \cap \xi$ and $t(\eta) \notin$ $V(t(\xi))$. Now, if $s, t \in T$, then let us set $s \preceq t$ iff $s \subseteq t$ and whenever $\xi \in \operatorname{dom}(s)$, and $\eta \in \operatorname{dom}(t)$ is $(\xi, t)$-bad, there is an $\eta^{\prime} \in \operatorname{dom}(s) \backslash \eta$ such that $\eta^{\prime}$ is $(\xi, t)$-bad.

One can easily check that $\preceq$ as defined above is a partial order on $T$. Also note that if $t \in T$ and $\zeta \in \omega_{1}$, then $t \mid \zeta \in T$ and $t \mid \zeta \preceq t$.

The author does not know whether $\langle T, \preceq\rangle$ itself is a proper poset. As explained in [T, p. 212], there is no reason to believe so, and one has to introduce a new poset $P$ that will consist of members of $T$ augmented by "side conditions".

To set up $P$, let $\kappa \geq \omega_{1}$ be an ordinal so big that $X$ belongs to $V_{\kappa}$. Then define

$$
\begin{array}{r}
N=\left\{\langle x, \varphi, M\rangle: x \in X, M \in\left[V_{\kappa+\omega_{1}}\right]^{\leq \omega}, \varphi \text { is a function from } T \text { to } \mathscr{H}\right. \\
\text { such that } \left.x \notin \varphi(t) \text { for every } t \in T \cap[M]^{<\omega}\right\} \subset V_{\kappa+\omega_{1}} .
\end{array}
$$

If $p: \omega_{1} \rightarrow N$ is a partial function and for every $\xi \in \operatorname{dom}(p), p(\xi)=$ $\left\langle x_{\xi}, \varphi_{\xi}, M_{\xi}\right\rangle$, then define the functions $\hat{p}, V p, \varphi p, M p$ on $\operatorname{dom}(p)$ by $\hat{p}(\xi)=x_{\xi},(V p)(\xi)=V(\hat{p}(\xi)),(\varphi p)(\xi)=\varphi_{\xi},(M p)(\xi)=M_{\xi}$. Now let $P$ be the set of all finite partial functions $p$ from $\omega_{1}$ to $N$ satisfying the following conditions:

(D1) $\hat{p} \in T$;

(D2) if $\eta, \xi \in \operatorname{dom}(p), \eta<\xi$, then 
(a) $[(\varphi p)(\eta)](t) \subseteq[(\varphi p)(\xi)](t)$ for every $t \in T$;

(b) $(M p)(\eta) \cup\{\langle\eta, \hat{p}(\eta)\rangle,\langle\eta, p(\eta)\rangle\} \subseteq(M p)(\xi)$

For every $p \in P$, define $\tilde{\varphi}(p)$ and $\widetilde{M}(p)$ by:

[a] for every $t \in T, \tilde{\varphi}(p)(t)=[(\varphi p)(\zeta)](t)$ where $\zeta=\max \operatorname{dom}(p)$;

[b] $\widetilde{M}(p)=\bigcup\{(M p)(\xi) \cup\{\langle\xi, \hat{p}(\xi)\rangle,\langle\xi, p(\xi)\rangle\}: \xi \in \operatorname{dom}(p)\}$.

Now, if $p, q \in P$, then we shall say that $p \leq q$ iff $p \subseteq q$ and $\hat{p} \preceq \hat{q} . \leq$ is a partial order on $P$ since $\preceq$ was a partial order on $T$. Also note that $p \in P$ and $\zeta \in \omega_{1}$ imply $p \uparrow \zeta \in P$ and $p \uparrow \zeta \leq p$.

We have to prove that $P$ is proper. To begin with let us call a choice of a $p_{0} \in P$ and a sequence $\left\{A_{n}: n \in \omega\right\}$ of maximal up-antichains in $P$ a challenge. Given a challenge $p_{0},\left\{A_{n}: n \in \omega\right\}$ and an increasing sequence $\left\{P_{n}: n \in \omega\right\}$ of countable subsets of $P$, let us define $P^{*}=\bigcup\left\{P_{n}: n \in \omega\right\}$ and for every $n \in \omega$, let $A_{n}^{*}=\left\{p \in A_{n}\right.$ : there is a $q \in P^{*}$ with $\left.p \leq q\right\}$. We shall say that $\left\{P_{n}: n \in \omega\right\}$ is a proper answer to the challenge $p_{0},\left\{A_{n}: n \in \omega\right\}$ if the following condition holds:

(*) there exists a $p_{1} \geq p_{0}$ such that for every $m \in \omega$ and $r \geq p_{1}$, there is a $p_{2}$ in $A_{m}^{*}$ which is upwards-compatible with $r$.

By the game-theoretic characterization of a proper poset $\left[\mathrm{Ba}, \mathrm{Fr}_{1}\right]$, we are done if we can prove the following:

Claim. For every challenge $p_{0},\left\{A_{n}: n \in \omega\right\}$ we can assign a proper answer $\left\{P_{n}: n \in \omega\right\}$ in such a way that $P_{n+1}$ only depends on $p_{0}, A_{0}, \ldots, A_{n}$; i.e., whenever $p_{0},\left\{A_{n}: n \in \omega\right\}$ and $p_{0},\left\{A_{n}^{\prime}: n \in \omega\right\}$ are two challenges, $\left\{P_{n}: n \in\right.$ $\omega\},\left\{P_{n}^{\prime}: n \in \omega\right\}$ are the proper answers assigned to them, $n \in \omega$ and $A_{i}=A_{i}^{\prime}$ for every $i \leq n$, then $P_{n+1}=P_{n+1}^{\prime}$.

In order to see that the claim is true, let us start with a challenge $p_{0},\left\{A_{m}: m\right.$ $\in \omega\}$. For each $m \in \omega$ let $Q_{m}=\left\{q \in P\right.$ : there is a $p \in A_{m}$ with $q \geq$ $p\}$. Also, for every $p \in P, m \in \omega$ and $t \in T$ define $K_{k}(p, m) \subseteq T$ and $Z(p, m, t, k) \subseteq X$ by induction on $k \in \omega$, as follows:

$K_{0}(p, m)=\left\{t \in T:\right.$ there is a $q \in Q_{m}$ with $q \geq p$ and $\left.\hat{q}=t\right\} ;$

$Z(p, m, t, k)=\{x \in X:$ there is a countable ordinal $\xi>\sup (\operatorname{dom}(t))$

such that $\left.t \cup\{\langle\xi, x\rangle\} \in K_{k}(p, m)\right\}$;

$K_{k+1}(p, m)=\{t \in T: Z(p, m, t, k) \notin \mathscr{H}\}$.

By induction on $n \in \omega$ let us define $M_{n}, \delta_{n}, W_{n}$ in such a way that the following conditions hold for every $n \in \omega$ : (i) (a) $M_{n} \in\left[V_{\kappa+\omega_{1}}\right]^{\leq \omega}, \delta_{n} \in \omega_{1}$ and $W_{n} \in \mathscr{H}_{0}$;

(b) $M_{0}=\left\{p_{0}\right\} \cup \omega, \delta_{0}=\omega, W_{0}=\varnothing$;

(ii) (a) $M_{n+1} \supseteq\left[M_{n}\right]^{<\omega} \cup \delta_{n} \cup M_{n}$;

(b) every pair and every finite union of members of $M_{n}$ is contained in $M_{n+1}$; 
(c) if $p \in P \cap M_{n}$, then $\hat{p} \in M_{n+1}$, range $(\hat{p}), \widetilde{M}(p) \subseteq M_{n+1}$;

(d) if $q \in P$ and $q \subseteq M_{n}$, then $q \in M_{n+1}$;

(e) if $m \leq n, p \in P \cap M_{n}$ and $t \in K_{0}(p, m) \cap M_{n}$ then there is a $q \in$ $Q_{m} \cap M_{n+1}$ such that $q \geq p$ and $\hat{q}=t$

(f) if $L_{n}=\left\{\langle p, m, t, k\rangle \in M_{n}: p \in P, m \leq n, t \in T, k \in \omega\right.$ and $Z(p, m, t, k) \notin \mathscr{H}\}$, then $\bigcap\left\{\overline{M_{n+1} \cap Z(p, m, t, k)} \backslash W_{n}:\langle p, m, t, k\rangle \in L_{n}\right\}$ $\neq \varnothing$.

To see that (f) can be satisfied note that for every $l \in L_{n}, \overline{Z(l)} \backslash W_{n} \in$ $\mathscr{F}$. Since $\mathscr{F}$ is countably closed, there is a point $x \in \bigcap\left\{\overline{Z(l)} \backslash W_{n}: l \in L_{n}\right\}$. Since $X$ has countable tightness, for every $l \in L_{n}$ there is a set $Z(x, l) \in$ $[Z(l)]^{\leq \omega}$ such that $x \in \overline{Z(x, l)}$. Now in order for $x$ to be in the intersection $\bigcap\left\{\overline{M_{n+1} \cap Z(l)} \backslash W_{n}: l \in L_{n}\right\}$, we only have to make sure that $M_{n+1}$ contains $Z(x, l)$ for every $l \in L_{n}$. Since $L_{n}$ is countable, this can be done.

Let us then continue with the conditions on $\delta_{n+1}$ and $W_{n+1}$ given that $M_{n}, \delta_{n}, W_{n}$ have already been constructed:

(iii) (a) $\delta_{n+1}>\delta_{n}$;

(b) $\operatorname{dom}(p) \subseteq \delta_{n+1}$ for every $p \in P \cap M_{n}$;

(c) if $p \in P \cap M_{n}, t \in T \cap M_{n}, x \in X \cap M_{n}, m \leq n, k \in \omega$ and there is a $\xi \in \omega_{1}$ such that $\xi>\sup (\operatorname{dom}(t))$ and $t \cup\{\langle\xi, x\rangle\} \in K_{k}(p, m)$, then there is a $\xi \in \delta_{n+1}$ with the same properties;

(iv) (a) $W_{n+1} \supseteq W_{n}$;

(b) $A(x) \subseteq W_{n+1}$ for every $x \in X \cap M_{n}$;

(c) if $p \in P \cap M_{n}, t \in T \cap M_{n}$, then $\tilde{\varphi}(p)(t) \subseteq W_{n+1}$;

(d) if $p \in P \cap M_{n}, m \leq n, t \in T \cap M_{n}$ and $k \in \omega$, then $Z(p, m, t, k) \in \mathscr{H}$ implies $Z(p, m, t, k) \subseteq W_{n+1}$.

Having finished the induction above, $\left\{P \cap M_{n}: n \in \omega\right\}$ is the designed proper answer to the challenge $p_{0},\left\{A_{n}: n \in \omega\right\}$. To see that in this way we can make an assignment as required in the claim, note first that in the conditions (i)(iv) for $n+1$ we only referred to the initial segment $p_{0}, A_{0}, \ldots, A_{n}$ of the challenge. Thus for each challenge $p_{0},\left\{A_{n}: n \in \omega\right\}$ we can assign a sequence $\left\{\left\langle M_{n}, \delta_{n}, W_{n}\right\rangle: n \in \omega\right\}$ in such a way that $P_{n+1}=P \cap M_{n+1}$ only depends on $p_{0}, A_{0}, \ldots, A_{n}$.

It only remains to show that whenever $\left\{\left\langle M_{n}, \delta_{n}, W_{n}\right\rangle: n \in \omega\right\}$ satisfies (i)(iv), then $\left\{P \cap M_{n}: n \in \omega\right\}$ is a proper answer to the challenge $p_{0},\left\{A_{n}: n \in \omega\right\}$. To prove this, set $M^{*}=\bigcup\left\{M_{n}: n \in \omega\right\}, P^{*}=P \cap M^{*}, \delta^{*}=\sup \left\{\delta_{n}: n \in \omega\right\}$ and $W^{*}=\bigcup\left\{W_{n}: n \in \omega\right\}$.

From (i)-(iv) it immediately follows that the following conditions hold:

(G) (a) $M^{*} \in\left[V_{\kappa+\omega_{1}}\right]^{\leq \omega}, \delta^{*} \in \omega_{1}$ and $W^{*} \in \mathscr{H}_{0}$;

(b) $\left\{p_{0}\right\} \cup \delta^{*} \subseteq M^{*}, \omega<\partial^{*}$;

(M) (a) $M^{*}$ is closed under building pairs and taking finite unions; further, every finite subset of $M^{*}$ is an element of $M^{*}$;

(b) if $p \in P^{*}$, then $\hat{p} \in M^{*}$, range $(\hat{p}), \widetilde{M}(p) \subseteq M^{*}$;

(c) if $q \in P$ and $q \subseteq M^{*}$, then $q \in P^{*}$; 
(d) if $m \in \omega, p \in P^{*}$ and $t \in K_{0}(p, m) \cap M^{*}$, then there is a $q \in Q_{m} \cap M^{*}$ such that $q \geq p$ and $\hat{q}=t$;

(e) if $L=\bigcup\left\{L_{n}: n \in \omega\right\}=\left\{\langle p, m, t, k\rangle \in M^{*}: p \in P, t \in T, m, k \in \omega\right.$ and $Z(p, m, t, k) \notin \mathscr{H}\}$, then there is a point $y^{*} \in\left\{\overline{M^{*} \cap Z(p, m, t, k)} \backslash W^{*}\right.$ : $\langle p, m, t, k\rangle \in L\}$.

Again, let us stop to see that (e) can be satisfied. Since $X$ is countably compact it is enough to see that $\left\{\overline{M^{*} \cap Z(l)} \backslash W^{*}: l \in L\right\}$ is centered. So let $L^{\prime} \in[L]^{<\omega}$. We have to show that $Y=\bigcap\left\{\overline{M^{*} \cap Z(l)}: l \in L^{\prime}\right\} \nsubseteq W^{*}$. Suppose indirectly that $Y \subseteq W^{*}$. Then, since $Y$ is a closed subspace of a countably compact space, there is an $n \in \omega$ with $Y \subseteq W_{n}$. Without loss of generality assume $L^{\prime} \subseteq L_{n}$. Then $Y \supseteq \bigcap\left\{\overline{M_{n+1} \cap Z(l)}: l \in L_{n}\right\}$. By (ii)(f), the intersection on the right-hand side is not contained in $W_{n}$, in contradiction with $Y \subseteq W_{n}$.

Let us go on by listing the conditions for $\delta^{*}$ and $W^{*}$ :

(D) (a) $\operatorname{dom}(p) \subseteq \delta^{*}$ for every $p \in P^{*}$;

(b) if $p \in P^{*}, t \in T \cap M^{*}, x \in X \cap M^{*}, m, k \in \omega$ and there is a $\xi \in \omega_{1}$ such that $\xi>\sup (\operatorname{dom}(t))$ and $t \cup\{\langle\xi, x\rangle\} \in K_{k}(p, m)$, then there is a $\xi \in \delta^{*}$ with the same properties;

(W) (a) $A(x) \subseteq W^{*}$ for every $x \in X \cap M^{*}$;

(b) if $p \in P^{*}$ and $t \in T \cap M^{*}$, then $\tilde{\varphi}(p)(t) \subseteq W^{*}$;

(c) if $p \in P^{*}, t \in T \cap M^{*} ; m \in \omega$ and $k \in \omega$, then $Z(p, m, t, k) \in \mathscr{H}$ implies $Z(p, m, t, k) \subseteq W^{*}$.

Having the properties of $M^{*}, P^{*}, \delta^{*}, W^{*}$ listed we can turn to the proof that condition $(*)$ in the definition of a proper answer is satisfied. Some easy verifications will be left to the reader. In all of these cases the reader has to refer to one of the above properties which was part of the reason that these closedness properties have been explicitly listed.

We have to find a $p_{1} \geq p_{0}$ such as required in $(*)$. It will be a one-point extension of $p_{0}$ as follows. First, for every $t \in T$, set $\mathscr{Z}(t)=\{Z(p, m, t, k): p$ $\left.\in P^{*}, m, k \in \omega\right\} \cap \mathscr{H}$, and let $\psi^{*}(t)=\bigcup\left\{\tilde{\varphi}(p)(t): p \in P^{*}\right\} \cup \bigcup \mathscr{Z}(t) \cup W^{*} \in \mathscr{H}$.

Next take $y^{*}$ from $(\mathrm{M})(\mathrm{e})$ and let $p_{1}=p_{0} \cup\left\{\left\langle\delta^{*},\left\langle w^{*}, \psi^{*}, M^{*}\right\rangle\right\rangle\right\}$. The easy verification that $p_{1} \in P^{*}$ and that $p_{0}=p_{1} \mid \delta^{*} \leq p_{1}$, are left to the reader. The proof that $p_{1}$ is as required in $(*)$ will be based on the following crucial property of $\delta^{*}$ and $y^{*}$ :

(CP) if $m, k \in \omega, p \in P^{*}, t \in T,\left\langle\delta^{*}, y^{*}\right\rangle \in t, s=t \mid \delta^{*} \in K_{k}(p, m) \cap$ $M^{*}$, and $\xi \in \operatorname{dom}(t) \backslash \delta^{*}$ implies $t(\xi) \notin W^{*}$, then there is an $s^{\prime} \in K_{0}(p, m) \cap$ $M^{*}$ such that $t \cup s^{\prime} \in T, s \preceq s^{\prime}$ and $t \preceq t \cup s^{\prime}$.

We are going to prove (CP) by induction on $k \in \omega$. For $k=0$ take $s=s^{\prime}$. So assume that we have already proved (CP) for $k$, and suppose that the conditions of (CP) are satisfied for $k+1$. Then, in particular, $s=t\left\lceil\delta^{*} \in\right.$ $K_{k+1}(p, m)$, so $Z(p, m, s, k) \notin \mathscr{H}$. By $(\mathbf{M})(\mathrm{e}), y^{*} \in \overline{M^{*} \cap Z(p, m, s, k)}$, 
so there is a point $x \in M^{*} \cap Z(p, m, s, k) \cap U$, where

$$
\begin{aligned}
& U=\bigcap\left\{V(t(\xi)): \xi \in \operatorname{dom}(t) \text { and } y^{*}\right.\in V(t(\xi))\} \backslash \\
& \bigcup\left\{A(t(\xi)): \xi \in \operatorname{dom}(t) \cap \delta^{*}=\operatorname{dom}(s)\right\} .
\end{aligned}
$$

Note that $y^{*} \in U$ by $(\mathrm{W})(\mathrm{a})$. Since $x \in Z(p, m, s, k)$ there is an $\eta>$ $\sup (\operatorname{dom}(s))$ such that $s \cup\{\langle\eta, x\rangle\} \in K_{k}(p, m)$. By (D)(b) we may assume that $\eta \in \delta^{*}$. By $x \in M^{*} \cap U$ and $(\mathrm{W})(\mathrm{a}), t \cup\{\langle\eta, x\rangle\} \in T$. By (G)(b), $s, x \in M^{*}$ and $(\mathrm{M})(\mathrm{a}), s \cup\{\langle\eta, x\rangle\} \in M^{*}$.

It follows from the above that $m \in \omega, p \in P^{*}, s \cup\{\langle\eta, x\rangle\}$ and $t \cup\{\langle\eta, x\rangle\}$ satisfy the conditions of (CP) with $k$ instead of $k+1$. Thus by the induction hypothesis there is an $s^{\prime} \in K_{0}(p, m) \cap M^{*}$ such that $t \cup\{\langle\eta, x\rangle\} \cup s^{\prime} \in T$, $s \cup\{\langle\eta, x\rangle\} \preceq s^{\prime}$ and $t \cup\{\langle\eta, x\rangle\} \preceq t \cup\{\langle\eta, x\rangle\} \cup s^{\prime}=t \cup s^{\prime}$.

Now observe that $t \preceq t \cup\{\langle\eta, x\rangle\}$. This is, because if $\xi \in \operatorname{dom}(t)$ and $\eta$ is $(\xi, t \cup\{\langle\eta, x\rangle\})$-bad, then by the definition of $U, \delta^{*}$ is $(\xi, t \cup\{\langle\eta, x\rangle\})-$ bad, too. Thus $s^{\prime} \in K_{0}(p, m) \cap M^{*}, t \cup s^{\prime} \in T, s \preceq s \cup\{\langle\eta, x\rangle\} \preceq s^{\prime}$ and $t \preceq t \cup\{\langle\eta, x\rangle\} \preceq t \cup s^{\prime}$, and the induction proceeds.

Finally, to prove (*) let $m \in \omega, r \geq p_{1}$, and let $r^{\prime}=r \mid \delta^{*} \in P$. Without loss of generality we may assume that $r \in Q_{m}$. By $\left\langle\delta^{*},\left\langle w^{*}, \psi^{*}, M^{*}\right\rangle\right\rangle \in r$ and (D2)(b) in the definition of $P, r^{\prime} \subseteq M^{*}$. Thus by (M)(c), $r^{\prime} \in P^{*}$. Let $\delta^{*}=\gamma_{0}<\gamma_{1}<\cdots<\gamma_{n-1}$ enumerate $\operatorname{dom}(r) \backslash \delta^{*}$. For every $i<n$, let $r\left(\gamma_{i}\right)=\left\langle y_{i}, \psi_{i}, M_{i}^{*}\right\rangle$ and for every $j \leq n$, let $r_{j}=r \uparrow\left(\delta^{*} \cup\left\{\gamma_{i}: i<j\right\}\right)$. As is usual in such proofs of properness we are going to induce backwaras on the length of $r_{j}$ to prove that

(B) for every $k \leq n, \hat{r}_{n-k} \in K_{k}\left(r^{\prime}, m\right)$.

If $k=0$, then $\hat{r}_{n}=\hat{r} \in K_{0}\left(r^{\prime}, m\right)$ trivially. Suppose that we are done for $k-1 \geq 0$. Then $\hat{r}_{n-(k-1)}=\hat{r}_{n-k} \cup\left\{\left\langle\gamma_{n-k}, y_{n-k}\right\rangle\right\} \in K_{k-1}\left(r^{\prime}, m\right)$. Thus $y_{n-k} \in Z\left(r^{\prime}, m, \hat{r}_{n-k}, k-1\right)$. On the other hand, from (D2)(b) in the definition of $P$ it follows that $\hat{r}_{n-k} \in\left[M_{n-k}^{*}\right]^{<\omega}$, so by $\left\langle y_{n-k}, \psi_{n-k}, M_{n-k}^{*}\right\rangle \in$ $N, y_{n-k} \notin \psi_{n-k}\left(\hat{r}_{n-k}\right) \supseteq \psi^{*}\left(\hat{r}_{n-k}\right) \supseteq \bigcup \mathscr{Z}\left(\hat{r}_{n-k}\right)$. Comparing this to $y_{n-k} \in Z\left(r^{\prime}, m, \hat{r}_{n-k}, k-1\right)$ it follows from the definition of $\mathscr{Z}(t)$ that $Z\left(r^{\prime}, m, \hat{r}_{n-k}, k-1\right) \notin \mathscr{H}$. Thus $\hat{r}_{n-k} \in K_{k}\left(r^{\prime}, m\right)$, and the induction proceeds.

In particular, taking $k=n$ in (B), it follows that $\hat{r}^{\prime} \in K_{n}\left(r^{\prime}, m\right)$. Also note that for each $i<n, \hat{r}\left(\gamma_{i}\right) \notin \psi_{i}\left(\hat{r}_{i}\right) \supseteq \psi^{*}\left(\hat{r}_{i}\right) \supseteq W^{*}$. Thus we can apply the crucial property (CP) to conclude that there is an $s^{\prime} \in K_{0}\left(r^{\prime}, m\right) \cap M^{*}$ such that $\hat{r} \cup s^{\prime} \in T, \hat{r}^{\prime} \preceq s^{\prime}$ and $\hat{r} \preceq \hat{r} \cup s^{\prime}$. By (M)(d) there is a $q \in Q_{m} \cap M^{*}$ such that $q \geq r^{\prime}$ and $\hat{q}=s^{\prime}$. Now let us consider $p=r \cup q$. It can easily be verified that $p \in P$. (In particular, (D2)(a) is satisfied since on the one hand, $q \geq r^{\prime}$, and on the other, $q \in P^{*}$ and so $\tilde{\varphi}(q)(t) \subseteq \psi^{*}(t) \subseteq \psi_{i}(t)$ for every $t \in T$ and $i<n$.) Further, since $q \in P^{*}, \operatorname{dom}(q) \subseteq \delta^{*}$ by (D)(a), so $q=p \mid \delta^{*} \leq p$. 
On the other hand, $r \leq p$ since $\hat{r} \preceq \hat{r} \cup s^{\prime}=\hat{p}$. Since $q \in Q_{m} \cap M^{*} \subseteq P^{*}$, there is a $p_{2} \in A_{m}^{*}$ with $p_{2} \leq q$. This proves $(*)$.

Having proved that $P$ is proper, observe that for every $\xi \in \omega_{1}, Q_{\xi}=\{p \in$ $P: \operatorname{dom}(p) \backslash \xi \neq \varnothing\}$ is dense in $P$. To see this, let $p \in P$ be arbitrary. If $\sup \operatorname{dom}(p)>\xi$, we are done; otherwise take any $\zeta>\xi$. There is a point

$$
x \notin \bigcup\{A(\hat{p}(\eta)): \eta \in \operatorname{dom}(p)\} \cup \bigcup\left\{\tilde{\varphi}(p)(t): t \in T \cap[\widetilde{M}(p)]^{<\omega}\right\},
$$

since the right-hand side belongs to $\mathscr{H}$. Then $q=p \cup\{\langle\zeta,\langle x, \tilde{\varphi}(p), \widetilde{M}(p)\rangle\rangle\} \in$ $Q_{\xi}$ and $p=q\lceil\zeta \leq q$.

Thus by PFA, there is an $\omega_{1}$-sequence $\left\{p_{\xi}: \xi \in \omega_{1}\right\}$ of pairwise compatible members of $P$ with distinct domains. By a standard $\Delta$-system argument we may assume that $\operatorname{dom}\left(p_{\xi}\right)=d_{\xi} \cup \Delta\left(\xi \in \omega_{1}\right)$ in such a way that

(1) $\xi \in \omega_{1}$ implies $d_{\xi} \neq \varnothing$;

(2) $\eta \in \xi \in \omega_{1}$ imply $\max \Delta<\min d_{\eta}<\max d_{\eta}<\min d_{\xi}$.

For each $\xi \in \omega_{1}$, let $x_{\xi}=\hat{p}_{\xi}\left(\min d_{\xi}\right)$. Then the definition and compatibility of the $p_{\xi}$ 's imply that $\left\{x_{\xi}: \xi \in \omega_{1}\right\}$ is an increasing $\omega_{1}$-sequence for the pair $\langle A, V\rangle$.

\section{APPLICATIONS OF THE THEOREM ON THE EXISTENCE OF INCREASING $\omega_{1}$-SEQUENCES}

Theorem 2.1 (PFA). In a compact Haudsorff space $X$ of countable tightness, every countably compact subspace is compact.

Proof. Suppose indirectly that there is a countably compact, noncompact subspace $Y$ of $X$. Then there is a free closed filter $\mathscr{F}$ in $Y$. Since $\mathscr{F}$ is free, for every $y \in Y$, there are, in $X$, open neighborhoods $G(y)$ and $U(y)$ of $y$ such that

(1) $\operatorname{cl}_{X}(G(y)) \cap Y$ is $\mathscr{F}$-small and

(2) $\operatorname{cl}_{X}(U(y)) \subseteq G(y)$ hold.

For every $y \in Y$, let $A(y)=\operatorname{cl}_{X}(G(y)) \cap Y$ and $V(y)=U(y) \cap Y$.

By Theorem 1.1, there is an increasing $\omega_{1}$-sequence $\left\{y_{\xi}: \xi \in \omega_{1}\right\} \subseteq Y$ for the pair $\langle A, V\rangle$. Then for every $\xi \in \omega_{1}$,

$$
X_{\xi}=\operatorname{cl}_{X}\left(\left\{y_{\eta}: \eta \leq \xi\right\}\right) \subseteq \operatorname{cl}_{X}\left(V\left(y_{\xi}\right)\right) \subseteq G\left(y_{\xi}\right) \subseteq X-\left\{y_{\sigma}: \sigma>\xi\right\} .
$$

Thus $\left\{x_{\xi}: \xi \in \omega_{1}\right\}$ is a strictly increasing sequence of compact subsets of $X$. Since $X$ has countable tightness, $X^{*}=\bigcup\left\{X_{\xi}: \xi \in \omega_{1}\right\}$ is compact. On the other hand, $\left\{G\left(y_{\xi}\right): \xi \in \omega_{1}\right\}$ is an open cover of $X^{*}$ with no finite subcover. This contradiction proves Theorem 2.1.

Theorem 2.2 (PFA $+\mathrm{c}=\omega_{2}$ ). Every compact Hausdorff space of countable tightness is sequential.

Proof. By a result of [IN], if MA holds, then a compact Hausdorff space is sequential iff every countably compact subspace is compact. Since PFA $+c=\omega_{2}$ implies MA, Theorem 2.1 is applicable. 
Remark. The referee pointed out that $c=\omega_{2}$ in the above theorem can be weakened to $2^{\omega}<2^{\omega_{2}}$.

Theorem 2.3 (PFA). If $X$ is a compact Hausdorff space of countable tightness, then every nonisolated point $x$ in $X$ has a nontrivial sequence converging to it.

Proof. Suppose $x \in X$ is a counterexample. Then $X \backslash\{x\}$ is countably compact, so by Theorem 2.1 it is compact, in contradiction with our assumption that $x$ was nonisolated.

By, in essence, a result of Burke [Bu] and Gruenhage [G] a compact Hausdorff space has countable tightness if and only if it does not contain a perfect preimage of the ordinal space $\omega_{1}$ (see in [Ny]). Thus Theorem 2.2 may be rephrased as

Theorem $2.4\left(\mathrm{PFA}+\mathrm{c}=\omega_{2}\right)$. A compact Hausdorff space is either sequential or else contains a perfect preimage of $\omega_{1}$.

D. Fremlin [ $\left.\mathrm{Fr}_{1}\right]$ gave an array of results under PFA concerning the problem when a closed preimage of $\omega_{1}$ contains a homeomorphic copy of $\omega_{1}$ itself. These results can be plugged into Theorem 2.4 to strengthen the conclusion for various classes of spaces. Here we shall make use of a strengthening of Fremlin's result observed by the author. The proof will appear in $\left[\mathrm{Fr}_{1}\right]$.

Theorem 2.5 ( PFA ). Every closed preimage of the ordinal space $\omega_{1}$ which has character $\leq \omega_{1}$, contains a copy of $\omega_{1}$.

For other consequences note that the result of Burke and Gruenhage referred to above also implies that the one-point compactification of a locally compact Hausdorff space has countable tightness if and only if it does not contain a perfect preimage of $\omega_{1}$, [B, Lemma 2.1]. Thus from Theorem 2.1 and 2.5 the following result follows.

Theorem 2.6 ( PFA). A locally compact, countably compact, noncompact Hausdorff space $X$ contains a perfect preimage of $\omega_{1}$. Moreover, if $X$ also has character $\leq \omega_{1}$, then $X$ contains a copy of $\omega_{1}$.

Corollary 2.7 (PFA). A countably compact manifold is metrizable if and only if it does not contain a copy of $\omega_{1}$.

By an example of M. E. Rudin and P. Zenor [RZ], (see also in [ $\mathrm{Ny}_{1}$ ], PFA cannot be omitted even from Corollary 2.7.

Finally note that there have been a number of results on sequential compact Hausdorff spaces. By Theorem 2.2, PFA $+\mathrm{c}=\omega_{2}$ implies that these results also hold for countably tight compact Hausdorff spaces thereby answering several questions in the literature. Details of this and further applications will appear elsewhere. 


\section{REFERENCES}

[A 1 ] A. V. Arhangel' skii, A survey of recent advances in general topology, old and new problems, Proc. Internat. Congr. Math. (Nice, 1970), Vol. 2, Gauthier-Villars, Paris, 1971, pp. 19-26.

$\left[\mathrm{A}_{2}\right]$ _ property, Dokl. Akad. Nauk SSSR 203 (1972), 473-476.

[A 3 ] _ Structure and classification of topological spaces and cardinal invariants, Uspekhi Mat. Nauk. 33 no. 6 (1978), 29-84.

[B] Z. Balogh, Locally nice spaces under Martin's Axiom, Comment. Math. Univ. Carolin. 24 (1983), 63-87.

[Ba] J. Baumgartner, Applications of the proper forcing axiom, Handbook of Set-Theoretic Topology (K. Kunen and J. Vaughan, editors), North-Holland, 1984, pp. 913-960.

[Bu] D. Burke, Closed mappings, Surveys in General Topology (M. Reed, editor), Academic Press, 1980, pp. 1-32.

[F] V. V. Fedorchuk, Fully closed mappings and the compatibility of some theorems of general topology with the axioms of set theory, Mat. Sb. (N.S.) 99 (1976), 3-33.

[Fr 1 ] D. Fremlin, Perfect preimages of $\omega_{1}$ and the PFA, Preprint.

[Fr 2 ] _ Consequences of Martin's maximum, Preprint.

[FN] D. Fremlin and P. Nyikos, Countably tight, countably compact spaces, Preprint.

[G] G. Gruenhage, Some results on spaces having an ortho-base or a base of subinfinite rank, Topology Proc. 2 (1977), 151-159.

[IN] M. Ismail and P. Nyikos, On spaces in which countably compact subsets are closed, and hereditary properties, Topology Appl. 11 (1980), 281-292.

[K] K. Kunen, Set theory, North-Holland, Amsterdam-New York-Oxford, 1980.

[KV] K. Kunen and J. Vaughan (editors), Handbook of set-theoretic topology, North-Holland, Amsterdam-New York-Oxford, 1984.

[MM] R. C. Moore and S. G. Mrowka, Topologies determined by countable objects, Notices Amer. Math. Soc. 11 (1964), 554.

[Ny 1 ] P. Nyikos, The theory of nonmetrizable manifolds, Handbook of Set-Theoretic Topology, North-Holland, 1984, pp. 633-684.

$\left[\mathrm{Ny}_{2}\right]$ ] Forcing compact non-sequential spaces of countable tightness, Preprint.

$\left[\mathrm{Ny}_{3}\right] \ldots$, Handwritten manuscript.

[Ny 4 ] _ Progress on countably compact spaces, Proc. Sixth Prague Topology Sympos. held in 1986, Heldermann, Berlin, pp. 379-410.

[O] A. Ostaszewski, On countably compact, perfectly normal spaces, J. London Math. Soc. (2) 14 (1976), 505-516.

[RZ] M. E. Rudin and P. Zenor, A perfectly normal nonmetrizable manifold, Houston J. Math. 2 (1976), 129-134.

[Sz] Z. Szentmiklóssy, S-spaces and L-spaces under Martin's Axiom, Colloq. Math. Soc. János Bolyai, 23, Budapest, 1978, II, North-Holland, Amsterdam, 1980, pp. 1139-1145.

[T] S. Todorcevic, A note on the proper forcing axiom, Contemp. Math. Vol. 31, Amer. Math. Soc., Providence, R.I., 1984, pp. 209-218.

[TP] Topology Proc. 2 (1977), 679-685.

Department of Mathematics, Kossuth University, Debrecen, Pf. 12, H-4010 Hungary

Current address: Department of Mathematics and Statistics, Miami University, Oxford, $\mathrm{OH}$ 45056, U.S.A. 